Elsevier Editorial System(tm) for The Lancet Manuscript Draft

Manuscript Number: THELANCET-D-18-06788

Title: Improving outcomes with a little EFFORT

Article Type: Linked Comment

Corresponding Author: Professor Dileep N Lobo, MS, DM, FRCS, FACS, FRCPE Corresponding Author's Institution: Nottingham University Hospitals and University of Nottingham

First Author: Dileep N Lobo, MS, DM, FRCS, FACS, FRCPE

Order of Authors: Dileep N Lobo, MS, DM, FRCS, FACS, FRCPE 


\section{Improving outcomes with a little EFFORT}

Dileep N Lobo, DM, FRCS ${ }^{1,2}$

${ }^{1}$ Gastrointestinal Surgery, Nottingham Digestive Diseases Centre, National Institute for Health Research (NIHR) Nottingham Biomedical Research Centre, Nottingham University Hospitals NHS Trust and University of Nottingham, Queen's Medical Centre, Nottingham NG7 2UH, UK

${ }^{2}$ MRC/ARUK Centre for Musculoskeletal Ageing Research, School of Life Sciences, University of Nottingham, Queen's Medical Centre, Nottingham NG7 2UK, UK

Word count (excluding title page and references): 897

Key words: nutritional intervention; nutritional risk; medical inpatients; outcome;

complications; mortality; commentary

Funding: No external funding was obtained for this commentary

Provenance: Invited article

Declaration of interests: DNL has collaborated with one of the authors (Z Stanga) in the past and they have published 8 peer-reviewed papers together, the most recent being in 2010 . DNL has received unrestricted research funding from BBraun and speaker's honoraria from BBraun, Fresenius Kabi, Baxter and Shire for unrelated work.

\section{Address for correspondence:}

Professor Dileep N Lobo

Gastrointestinal Surgery

Nottingham Digestive Diseases Centre

Nottingham University Hospitals NHS Trust and University of Nottingham

E Floor, West Block

Queen's Medical Centre

Nottingham NG7 2UH, UK

Tel: +44-115-8231149

Fax: $+44-115-8231160$

E-mail: Dileep.Lobo@nottingham.ac.uk 
The adverse effect of weight loss on clinical outcome was documented over 80 years ago when Hiram Studley showed that, in patients undergoing surgery for perforated duodenal ulcer, postoperative mortality was 10 times greater in those who had lost more than $20 \%$ of their body weight preoperatively compared with those who had lost less. ${ }^{1}$ Similar, if less dramatic results were shown in medical patients. The potential importance of these observations was emphasized by a study from the 1990 s showing that $30 \%$ of 500 hospitalised patients had moderate to severe malnutrition on admission. ${ }^{2}$ of the patients who stayed in hospital for more than one week, $65 \%$ continued to lose weight, with only a few of the malnourished patients being referred for nutritional intervention. ${ }^{2}$ While most hospitals in the developed world provide food that can meet the patients' nutritional requirements, more than $40 \%$ of this food may left on the plate and wasted, resulting in the consumption of less than $80 \%$ of the recommended protein and energy (minimum $1800 \mathrm{kcal} / \mathrm{day}$ ) intake being consumed by patients capable of eating. ${ }^{3}$ The reasons for reduced food intake, especially in the older adult, are multifactorial and can stem from the belief of patients that poor appetite and intake are "to be expected" during hospitalisation, with many supposing that their appetite will return to normal after discharge. ${ }^{4}$ Other reasons include gastrointestinal symptoms, inactivity, depression or low mood, inflexibility of hospital systems, quality of the food, lack of motivation and the belief by both staff and patients that medical treatment is the main priority and that food is of secondary importance. ${ }^{4,5}$

Despite these studies on the prevalence and clinical effects of malnutrition, there has, until now, been a lack of high-quality evidence to support the value of nutritional treatment in medical patients. A meta-analysis of 22 randomised controlled trials with 3736 participants assessed the effects of nutritional support on outcomes in medical inpatients with 
malnutrition or at risk of malnutrition. ${ }^{6}$ Although the review showed that nutritional support increased protein and energy intake and body weight, there was little effect on clinical outcome in terms of mortality, hospital-acquired infections, length of stay and functional improvement, ${ }^{6}$ findings also supported by a Cochrane review. ${ }^{7}$ A study not included in these analyses showed that although a high-protein oral nutritional supplement containing betahydroxy-beta-methylbutyrate had no effect on the primary composite endpoint of 90-day post-discharge incidence of death or non-elective readmission when compared with placebo, it was associated with decreased mortality and improved indices of nutritional status during the period of observation. ${ }^{8}$

The Effect of early nutritional support on Frailty, Functional Outcomes and Recovery of malnourished medical inpatients Trial (EFFORT) ${ }^{9}$ is a well-designed, pragmatic, unblinded, multi-centre trial that aimed to test the hypothesis that providing patients who are found to be at nutritional risk (Nutritional Risk Screening 2002 score $^{10} \geq 3$ ) with individualised nutritional support would result in a better outcome than in those given the standard hospital diet without any further nutritional intervention.

Of the 5015 patients screened, 2088 were enrolled and 2028 were included in the final analysis. The patients had an average age of 72 years with more than $82 \%$ being 65 years or older. Those in the intervention group received a modest $290 \mathrm{kcal} /$ day energy and $10 \mathrm{~g}$ protein/day more than the control group. However, and more importantly, during their hospital stay, energy goals were reached in $79 \%$ and protein goals in $76 \%$ of the intervention group, compared with $54 \%$ and $55 \%$ respectively in the control group. The investigators were able to show that this intervention led to a significantly better outcome when the primary composite end point was assessed (adverse clinical outcome defined as all-cause mortality, intensive care unit admission, non-elective hospital readmission, major 
complications and decline in functional status at 30 days). In addition, mortality (7.2\% vs. 9.9\%) and functional decline at 30 days were significantly lower, and quality of life and improvement in activities of daily living significantly better in the intervention group. Interestingly, $91 \%$ of the intervention was provided with food adjustment, food fortification and oral nutritional supplements after and, perhaps more importantly, individualised dietitian input. Enteral and parenteral nutrition were used in only 8 and 12 patients, respectively in the intervention group. The effect of nutritional support on the risk for the primary endpoint was consistent across predefined subgroups (except in those with chronic kidney disease, where the effect of nutritional support was more profound). This is an important study that has shown that a relatively simple intervention in patients at nutritional risk admitted to medical wards can result in significant improvements in outcome, with a need to treat 25 patients to prevent one adverse clinical outcome and 37 to prevent one death. What cannot be measured in this study is the contribution to outcome made by the regular visits of the dietician and the resulting encouragement and attention to detail in the intervention group. Nevertheless, these results are of general importance and support a change in clinical practice in which greater attention is paid to nutritional care in hospital.

EFFORT ${ }^{9}$ has provided $21^{\text {st }}$ century evidence to substantiate the aphorism of Hippocrates of Kos from the $4^{\text {th }} / 5^{\text {th }}$ century BC: "The patient ought likewise to be consider'd, whether he is able to hold out with the prescribed diet, even in the height of the disease; for if the diet is not sufficient, the patient will grow too faint, and be overcome by the disease."11 


\section{References}

1. Studley HO. Percentage of weight loss: a basic indicator of surgical risk in patients with chronic peptic ulcer. JAMA 1936; 106: 458-60.

2. McWhirter JP, Pennington CR. Incidence and recognition of malnutrition in hospital. BMJ 1994; 308: 945-8.

3. Barton AD, Beigg CL, Macdonald IA, Allison SP. High food wastage and low nutritional intakes in hospital patients. Clin Nutr 2000; 19: 445-9.

4. Hope K, Ferguson M, Reidlinger DP, Agarwal E. "I don't eat when I'm sick": older people's food and mealtime experiences in hospital. Maturitas 2017; 97: 6-13.

5. Hartwell HJ, Shepherd PA, Edwards JSA, Johns N. What do patients value in the hospital meal experience? Appetite 2016; 96: 293-8.

6. Bally MR, Blaser Yildirim PZ, Bounoure L, Gloy VL, Mueller B, Briel M, et al. Nutritional support and outcomes in malnourished medical inpatients: a systematic review and meta-analysis. JAMA Intern Med 2016; 176: 43-53.

7. Feinberg J, Nielsen EE, Korang SK, Halberg Engell K, Nielsen MS, Zhang K, et al. Nutrition support in hospitalised adults at nutritional risk. Cochrane Database Syst Rev 2017; 5: CD011598.

8. Deutz NE, Matheson EM, Matarese LE, Luo M, Baggs GE, Nelson JL, et al.; NOURISH Study Group. Readmission and mortality in malnourished, older, hospitalized adults treated with a specialized oral nutritional supplement: a randomized clinical trial. Clin Nutr 2016; 35: 18-26.

9. Schuetz P, Fehr R, Baechli V, Geiser M, Deiss M, Gomes F, et al. Individualized nutritional support in medical inpatients at nutritional risk: a randomized clinical trial. Lancet 2018 (In press). 
10. Kondrup J, Rasmussen HH, Hamberg O, Stanga Z; Ad Hoc ESPEN Working Group. Nutritional risk screening (NRS 2002): a new method based on an analysis of controlled clinical trials. Clin Nutr 2003; 22: 321-36.

11. Sprengell C. The Aphorisms of Hippocrates, and the Sentences of Celsus; with Explanations and References. London: R Wilkin, J and J Bonwick, 1735. 